Journal of Case Reports 2018;8(2):95-97

\title{
Femur Metastasis: A Rare Metastatic Site in Carcinoma Cervix
}

\author{
Purnima Thakur ${ }^{1}$, Vishal Verma ${ }^{2}$, Vikas Fotedar ${ }^{1}$, Mukesh Sharma $^{1}$ \\ Departments of ${ }^{1}$ Radiation Oncology, ${ }^{2}$ Orthopedics; Indira Gandhi Medical College, Shimla, Himachal Pradesh, India.
}

Corresponding Author:

Dr. Purnima Thakur

Email: purnimathakur28@gmail.com

This is an Open Access article distributed under the terms of the Creative Commons Attribution License (creativecommons.org) licenses/by/3.0).

Received : October 26, 2017

Accepted : March 13, 2018

Published : April 15, 2018

\begin{abstract}
Background: Cancer cervix is known to spread to distant sites including bones. Long bone metastasis is relatively rare and only handful of cases have been reported. Therefore, bone scan is routinely used in staging of cancer cervix. We report a rare case of cancer cervix with controlled primary and isolated metastasis to femur. Case Report: A 45 year female patient of cancer cervix IIB was treated by chemo-radiotherapy to pelvis and para-aortic region. On follow up, she developed isolated bone lesion in upper $1 / 3^{\text {rd }}$ of femur. Femoral involvement was histologically proven squamous cell carcinoma, consistent with the primary pathology. She was treated by surgical resection of metastatic disease with total hip replacement, followed by adjuvant chemotherapy. Presently she is on follow up for 10 months and asymptomatic. Conclusion: Bony metastasis may reduce the survival significantly and therefore early diagnosis and adequate treatment may lead to better quality of life in these patients.
\end{abstract}

Keywords: Arthroplasty, Chemotherapy, Femur, Squamous Cell Carcinoma, Uterine Cervical Neoplasms.

\section{Introduction}

Cancer cervix is known to spread commonly to lung, para-aortic nodes, the abdominal cavity and supra-clavicular nodes. Bone metastasis in cervical cancer is relatively rare and tends to predominantly involve the axial skeleton. The rate of bone metastasis have been reported between $2-16 \%[1,2,3]$. Long bone metastasis from cervix is still rare and only one other case of isolated femur metastasis has been reported [4]. We present a rare case of cancer cervix with solitary metastasis to femur.

\section{Case Report}

45 years female patient was diagnosed as a case of cancer cervix IIB moderately differentiated squamous cell carcinoma. Computerised tomography (CT) abdomen and pelvis showed heterogeneously enhancing mass measuring $3.5 \times 3.7 \mathrm{~cm}$ involving cervix and lower half of uterus. Mass was extending into upper $1 / 3^{\text {rd }}$ of vagina, with maintained fat planes with urinary bladder and rectum. Multiple lymphadenopathy was seen in bilateral external iliac, pre-aortic, para-aortic, largest measuring $18 \times 20 \mathrm{~mm}$. Bony structures appeared normal. She was treated with chemo-radiotherapy (CRT) at $50 \mathrm{~Gy} / 25$ fractions/5 weeks to pelvis and $45 \mathrm{~Gy} / 20$ fractions/4 weeks to para-aortic nodes with weekly concurrent injection cisplatin $40 \mathrm{mg} / \mathrm{m}^{2}$. Post CRT, she was not fit for intra-cavitory brachytherapy due to bulky parametrium (upto medial $2 / 3^{\text {rd }}$ ), therefore, she was further treated with supplement external beam radiation therapy (EBRT) to pelvis at $20 \mathrm{~Gy} / 10$ fractions/2 weeks with weekly cisplatin injection. On examination after 6 weeks of completion of CRT, there was no evidence of local disease and was kept on two monthly follow up. 10 months after completion of CRT, she complained of right thigh pain which was moderately severe, dull aching and continuous. X-ray femur showed lytic 
lesion in right upper femur shaft. Whole body scintigraphy bone scan revealed intense tracer uptake in proximal $1 / 3^{\text {rd }}$ of right femur shaft and head of right femur, with suspicion of metastatic disease. No other bone was affected. There was no evidence of local disease clinically. MRI pelvis showed no evidence of disease in cervix and bony lesion in proximal shaft and head of right femur with normal soft tissue structures. No lung or liver metastasis was detected on CT chest and abdomen. The patient underwent a CT guided biopsy of the lesion in the bone which showed metastatic squamous cell carcinoma consistent with her original cervical tumor.

In view of solitary metastatic lesion, total hip replacement was done. Detailed histo-pathological examination was suggestive of metastatic squamous cell carcinoma femur with invasion into soft tissue distal resection margin. In view of soft tissue involvement, she was given adjuvant chemotherapy of paclitaxel, cisplatin and 5-flouro uracil every 3 weekly. Total six courses were given and she was kept on follow up. Presently, she is on two monthly follow up since 10 months with no evidence of recurrence or metastasis.

\section{Discussion}

Carcinoma cervix is the most common cancer prevalent in Indian women. Women in this Himalayan region present in locally advanced stages. Many factors contribute to distant metastasis including advanced stage, endometrial extension and local failure [5]. Apart from spread to pelvic and para-aortic nodes, disease frequently spreads to lungs and liver. Although spread to bones is often seen in clinical practice, but long bone metastasis is a rare finding.

Bone metastasis may reduce survival and cause suffering in form of severe pain and pathological fractures. Early diagnosis and effective treatment could lead to a better quality of life and improved survival in these patients. Osteolytic lesions invariably present with bone

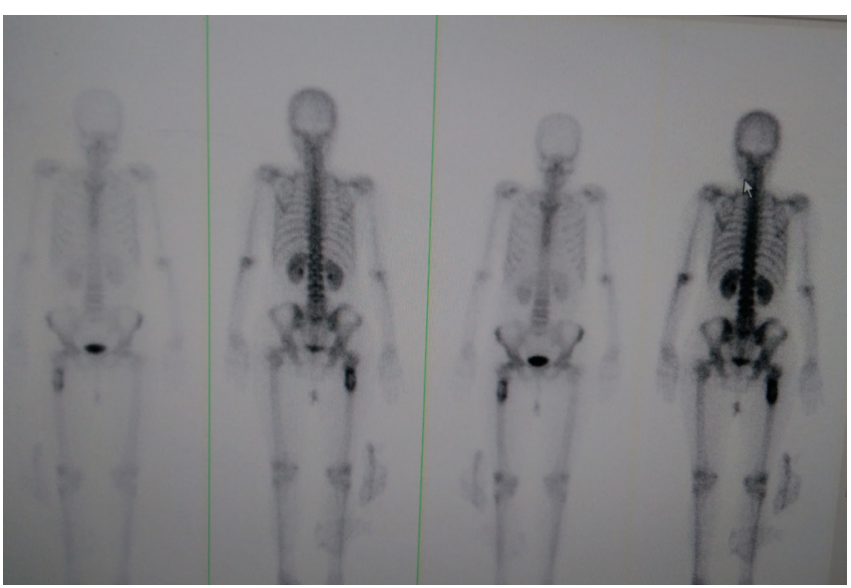

Fig.1: Bone scan AP/PA view showing tracer uptake in right upper femur shaft.

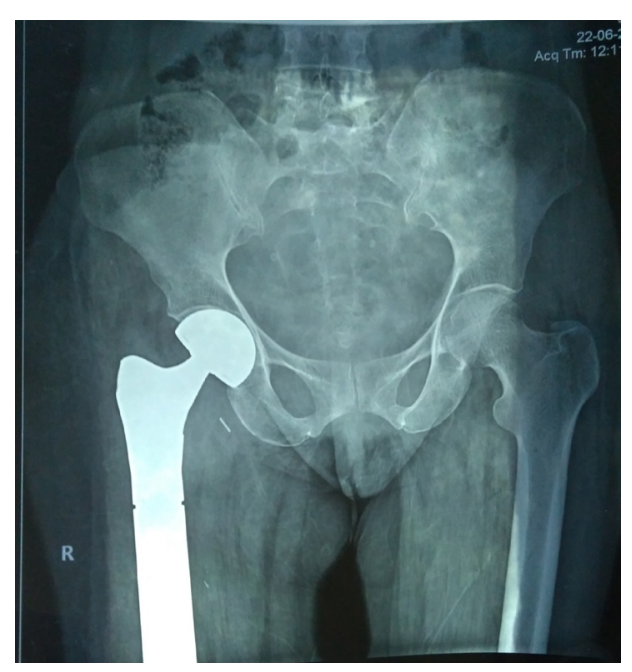

Fig.2: X-ray pelvis AP view (post total right hip replacement).

pain, or with associated pathologic fracture [6]. X-ray and magnetic resonance imaging show osteolytic lesions, and features may be confused with osteomyelitis. Few studies have evaluated the contributing factors and prognosis of bone metastasis. In a study of 105 patients of bone metastasis, adenocarcinoma, advanced stage (IIBIV) and initial multiple metastatic sites contribute to earlier bone metastasis [1]. Number of bone metastases and location of involved bone, were not associated with the overall survival of the patients.

Bony metastasis may occur via direct extension, lymph node metastases, spread through 
Batson plexus to spine, and hematogenous metastases, accounting for metastases to distant bones, and likely the mechanism of spread in this case. Most common sites of bony metastasis in cancer cervix are lumbar spine and pelvis, which get involved by direct and lymphatic spread [3]. Long bone metastasis attributable to hematogenous disease is rare. Lungs are the most common site of hematogenous spread and are involved in a patient presenting with bony metastasis [7]. 75-80\% of patients are lost within one year of diagnosis with bony metastases due to pulmonary or other complications $[7,8]$.

This is one such case of isolated long bone metastasis and with controlled primary disease. This case highlights the importance of recognition of this site of spread. Femur metastases are so uncommon that only one other source mentions them [4]. This case is valuable, because it highlights that even with controlled primary, the disease can reoccur at a distant site. There may be role of adjuvant chemotherapy after completion of local CRT in locally advanced cancer cervix to prevent such recurrence.

It is important to assess the patient's prognosis and other metastatic sites prior to offering further treatment. In our literature review, only one case study described a disease-free survival of over four years following resection of an isolated fibular metastasis in a patient of cancer cervix [5]. Survival in this case may be improved by offering wise and well planned treatment. Surgical resection was the primary treatment offered to such few patients reported in literature including ours. There is no proven role of radiotherapy to treat femur metastasis.

\section{Conclusion}

Due to rarity of long bone metastasis in cancer cervix, no specific treatment guidelines have been defined. Surgery, radiotherapy and chemotherapy remain the available options which can be used according to the clinical setting. However, despite the availability of these modalities, the prognosis remains poor. The aim of treatment in such cases should be to provide best palliation for pain and to maintain good quality of life as cases with solitary metastasis may survive for a longer duration.

Contributors: PT: manuscript writing, patient management; VV, VF: manuscript editing, patient management; MS: critical inputs into the manuscript. PT will act as guarantor. All authors approved the final version of this manuscript. Funding: None; Competing interests: None stated.

\section{References}

1. Yoon A, Choi CH, Kim HJ, Park JY, Lee YY, Kim TJ, et al. Contributing factors for bone metastasis in uterine cervical cancer. Int J Gynecol Cancer. 2013;23:13111317.

2. Barmeir E, Langer O, Levy JL, Nissenbaum M, DeMoor $\mathrm{NG}$, Blumenthal NJ. Unusual skeletal metastases in carcinoma of the cervix. Gynecol Oncol. 1985;20:307316.

3. Dewdney A, Selvarajah U. A 'hot' leg: a rare case of isolated long bone metastases from cervical cancer. Anticancer Res. 2010;30:2949-2951.

4. Corrado G, Santaguida S, Zannoni G, Scambia G, Ferrandina G. Femur metastasis in carcinoma of the uterine cervix: a rare entity. Arch Gynecol Obstet. 2010;281:963-965

5. Pasricha R, Tiwari A, Aggarwal T, Lal P. Carcinoma of uterine cervix with isolated metastasis to fibula and its unusual behaviour, report of a case and review of literature. J Cancer Res Ther. 2006;2:79-81.

6. Blythe JG, Cohen MH, Buchsbaum HJ, Latourette HB. Bony metastases from carcinoma of cervix. Occurrence, diagnosis, and treatment. Cancer. 1975;36:475-484.

7. Matsuyama $\mathrm{T}$, Tsukamoto $\mathrm{N}$, Imachi $\mathrm{M}$, Nakano $\mathrm{H}$. Bone metastasis from cervix cancer. Gynecol Oncol. 1989;32:72-75.

8. Ratanatharathorn V, Powers WE, Steverson N, Han I, Ahmad K, Grimm J. Bone metastasis from cervical cancer. Cancer. 1994;73:2372-2379. 